\title{
A solution for measuring accurate reaction time to visual stimuli realized with a programmable microcontroller
}

\author{
Toshio Ohyanagi And Yasuhito Sengoku \\ Sapporo Medical University, Sapporo, Japan
}

\begin{abstract}
This article presents a new solution for measuring accurate reaction time (SMART) to visual stimuli. The SMART is a USB device realized with a Cypress Programmable System-on-Chip (PSoC) mixed-signal array programmable microcontroller. A brief overview of the hardware and firmware of the PSoC is provided, together with the results of three experiments. In Experiment 1, we investigated the timing accuracy of the SMART in measuring reaction time (RT) under different conditions of operating systems (OSs; Windows XP or Vista) and monitor displays (a CRT or an LCD). The results indicated that the timing error in measuring RT by the SMART was less than $2 \mathrm{msec}$, on average, under all combinations of OS and display and that the SMART was tolerant to jitter and noise. In Experiment 2, we tested the SMART with 8 participants. The results indicated that there was no significant difference among RTs obtained with the SMART under the different conditions of OS and display. In Experiment 3, we used Microsoft (MS) PowerPoint to present visual stimuli on the display. We found no significant difference in RTs obtained using MS DirectX technology versus using the PowerPoint file with the SMART. We are certain that the SMART is a simple and practical solution for measuring RTs accurately. Although there are some restrictions in using the SMART with RT paradigms, the SMART is capable of providing both researchers and health professionals working in clinical settings with new ways of using RT paradigms in their work.
\end{abstract}

Timing accuracy in measuring reaction times (RTs) on computer systems has been studied by many researchers. It is known that monitor displays, devices for responding to stimuli, operating systems (OSs) of the computers used, and software for presenting stimuli were major technical factors influencing the timing accuracy. Krantz (2000) has discussed some issues with regard to the presentation of stimuli on monitor displays. Wiens et al. (2004) compared display technologies for presenting brief pictures and reported that LCD and TFT displays had poor accuracy. Plant, Hammond, and Whitehouse (2003) tested response devices, including a sample set of mice, a standard keyboard, and an E-Prime Deluxe response box, and showed that even changing the mouse could have an effect on RT measurement. Shimizu (2002) investigated the response characteristics of PC keyboards by comparing keyboards and joystick inputs. Forster and Forster (2003) developed DMDX software for solving timing errors in measuring RTs. Plant, Hammond, and Turner (2004) reported that there was a delay in displaying a stimulus on the screen even if the software was carefully developed to synchronize with the refresh rate. McKinney, MacCormac, and Welsh-Bohmer (1999) and De Clercq, Crombez, Buysse, and Roeyers (2003) solved the problem of timing errors by using external hardware and software capable of detecting the onset of the stimulus on the screen to measure RTs accurately. Forster and Forster pointed out that the cost-benefit ratio of the solution by McKinney et al. might be high, since the solution required the user to purchase additional expensive hardware. The solution by De Clercq et al. required an additional two PCs other than the PC under test. Recently, some commercial hardware and software products that measure RTs with millisecond accuracy have been developed. The Vienna Test System provides a self-calibration option to ensure the precision of RTs (Häusler, Sommer, \& Chroust, 2007). The Black Box Toolkit is an external calibration system that can be used with other test systems, such as E-Prime and SuperLab, to correct measurements manually (Plant et al., 2004). These systems, however, were developed mainly for use in laboratory settings and would not be easy to use for health professionals working in clinical settings.

This article presents our new solution for measuring accurate RT (SMART). The SMART was realized with a Cypress Programmable System-on-Chip (PSoC) mixedsignal array microcontroller. We developed new firmware running on the PSoCs. We herein first explain the hardware and firmware of the SMART and then report three experiments using the SMART. The results indicate that the SMART is a simple and practical solution to accurately measure RTs in both laboratory and clinical set-

T. Ohyanagi, ohyanagi@sapmed.ac.jp 
tings. Conclusions and some restrictions of the current version of SMART are also presented.

\section{THE SMART}

The SMART was developed to measure RTs to visual stimuli with millisecond accuracy. As a result of our investigations of previous studies and findings in this field, we concluded that such a solution should be realized as independently as possible from the computer systems used for presenting visual stimuli. We found that the Cypress PSoC mixed-signal array had a high potential for realizing the solution by using a photo IC for visual range to detect the onset of stimuli and by using PSoC's touch-sensing technology to obtain responses from participants. This section explains the hardware and firmware of the SMART.

\section{Hardware}

Cypress Semiconductor Corporation has been developing the family of PSoC microcontrollers. The PSoC consists of an 8-bit CPU core, programmable analog and digital blocks, system resources, and programmable I/O ports. On the basis of the $\mathrm{PSoC}$, analog-to-digital converters (ADCs), amplifiers, counters, communications such as full-speed USB and inter-integrated circuit (I2C), and other analog or digital functions can be easily realized. One more unique feature of the $\mathrm{PSoC}$ is its capability of capacitive touch sensing (CapSense) that can be replaced with mechanical buttons, switches, sliders, and proximity detectors already used in many industrial applications.

We implemented the SMART by attaching an S7565 photodiode (Hamamatsu Photonics K. K., 2001) and some electronic parts to a CY3270 PSoC FirstTouch Kit (Cypress Semiconductor Corporation, 2007). The S7565 is a linear current amplification photo IC for visual range. It is used to detect stimulus onset on the display. The kit consists of a USB interface dongle and a multifunction expansion card. The expansion card connects to the dongle through the dongle's $8 \times 2$ pin expansion port. A CY8C24894 PSoC, residing on the dongle, performs all of the USB and expansion card interface functions. The CY8C21434 PSoC and a variety of peripheral components that facilitate experiments with many different sensors and signal types reside on the expansion card. The sensors embedded therein are an ambient light sensor, a temperature sensor, the CapSense 7-element touch slider, and the CapSense proximity sensor. Each of the sensors is connected to ascribed PSoC I/O pins. Some registers, capacitors, and LEDs are also embedded in the card.

Figure 1A shows a schematic of the SMART. The ambient light sensor was replaced with the photodiode, and a condenser of $10 \mu \mathrm{F}$ was added to reduce the influence of noise on its signal. The port of proximity antenna, indicated as PRX1 in Figure 1A, was used to connect an electrode made of copper foil to sense touch. The SMART is a USB device, and its power is supplied by the computer system through the USB connection. Figure 1B shows a photo of the SMART. The cost of the hardware was about \$40, including the Cypress CY3270.

\section{Firmware}

The firmware for each PSoC was developed using PSoC Designer 5.0 and PSoC Programmer 3.0 software provided by Cypress.
A

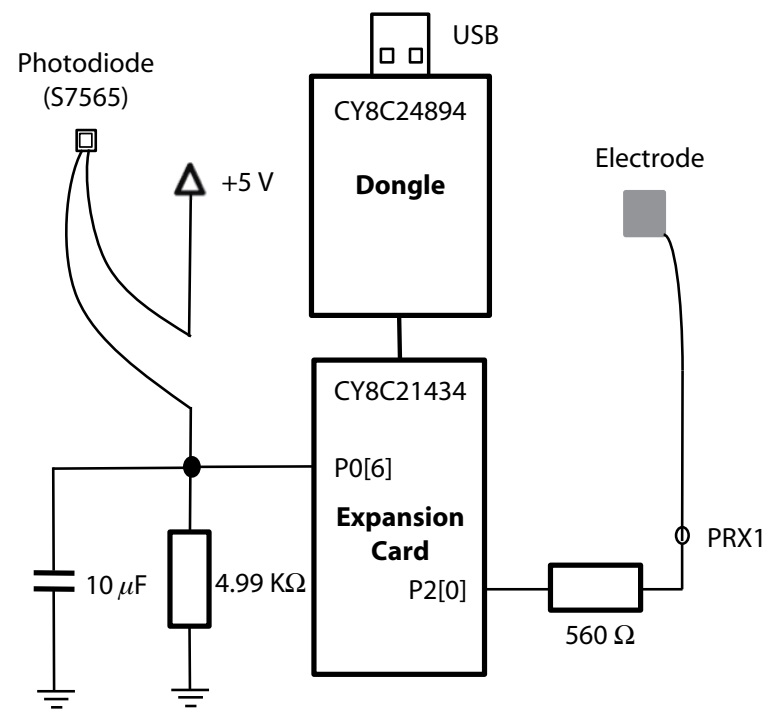

B

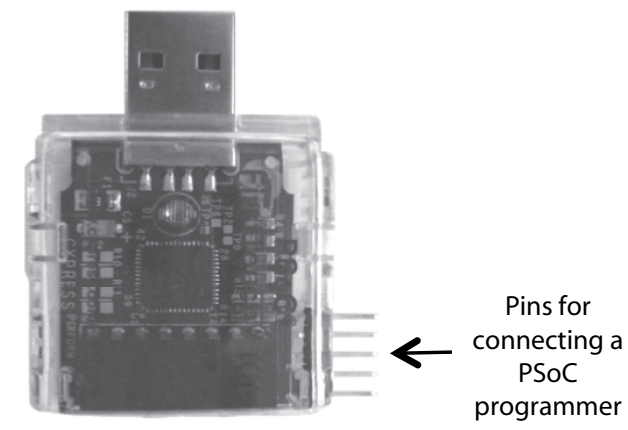
Expansion
Card

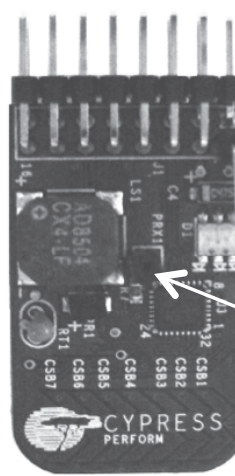

$10-\mu \mathrm{F}$

condenser

Figure 1. (A) Schematic of the SMART. The light sensor was embedded in a tiny housing of $10 \times 10 \times 8 \mathrm{~mm}$ and was attached to the left center of the monitor display. The electrode was put on the left button of the mouse (see Figure 3). (B) Photo of the SMART. 


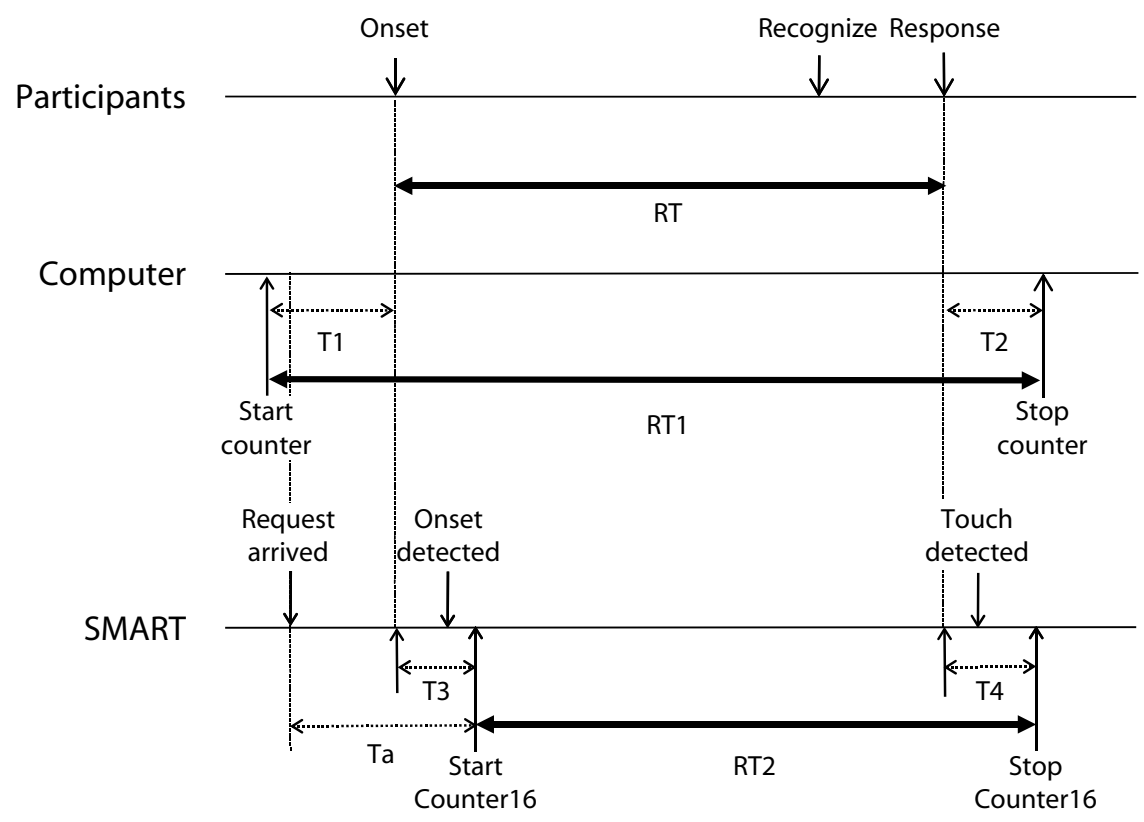

\begin{abstract}
Figure 2. Timing errors in measuring reaction time (RT) by a computer and the SMART. To measure RT1, the QueryPerformanceCounter function was used at the times of "Start counter" and "Stop counter." There are timing errors, T1 and T2, in RT1. T1 is a delay from the instruction to present a stimulus to the actual stimulus onset. $\mathrm{T} 2$ is a delay from an actual buttonpress to its registration in the computer. RT2 was measured by using user module Counter16 of the PSoC. T3 and T4 are latencies in starting and stopping the Counter16, respectively. Ta is time between arrival of the request from the PC to the dongle and the starting time of Counter 16.
\end{abstract}

On the PSoC of the dongle, we placed a Counter16 user module with a clock of $10 \mathrm{KHz}$, a USBFS user module, an I2CHW user module, and an LED user module. The Counter16 was used to measure RT with $0.1-\mathrm{msec}$ accuracy, and the USBFS was used to make the SMART a mechanism of the USB human interface device (HID) class. The I2CHW allowed communication with the expansion card.

On the PSoC of the expansion card, we placed an I2CHW user module and an ADC10 user module or a CSD user module. The ADC10 was used for converting analog data from the photodiode to digital. The converted digital value is used for judging the onset of stimuli on a display. The CSD was used for detecting touch to the electrode. Because both the ADC10 and the CSD could not be placed on the PSoC together, due to its limitation of resources, a dynamic reconfiguration technique was employed to switch from the ADC10 to the CSD and vice versa.

Flowcharts of the firmware for the PSoCs are shown in Appendix A. The dongle is for measuring RT and returning the measured RT to the computer system. The dongle starts the Counter16 after getting notification of the stimulus onset from the expansion card and stops it immediately after receiving notification of touch to the electrode from the card. This measurement is done independently of the computer system. The expansion card starts the ADC10 first. It then waits for a request from the dongle, and upon that request, it checks the onset of a stimulus on the display. After detecting the onset, the expansion card notifies the dongle of this, and thereafter the expansion card works to check touch to the electrode by using the CSD. After detecting the touch, the CSD is stopped, and the ADC is started again; then the dongle is notified of this process by the card.

\section{EXPERIMENT 1}

Figure 2 shows a timing chart of RT measurement by a computer and the SMART. T1 is a delay from an instruction to present a stimulus executed in a computer to the actual stimulus onset. T2 is a delay from an actual buttonpress to its registration in the computer. $\mathrm{T} 3$ and $\mathrm{T} 4$ are latencies in starting and stopping the Counter16 after the actual stimulus onset and touch, respectively. To investigate timing errors $\mathrm{T} 1$ and $\mathrm{T} 2$ and the timing accuracy of the SMART, we conducted the following experiment.

\section{Method}

Apparatus. A laptop PC (Model nw8000 from HP), a CRT display (Model FlexScan 54T from Nanao), and an LCD display (Model RDT176S from Mitsubishi) were used for this experiment. The PC was set up to select Windows XP SP3 or Windows Vista SP1 at boot time. We used 17-in. monitor displays, and their refresh rates were set at 100 and $60 \mathrm{~Hz}$ for $54 \mathrm{~T}$ and RDT176S displays, respectively. An S7565 photodiode was attached to the left center of the display. An MS serial mouse whose FCC ID was C3KSS1 was used to get responses. The mouse ball was removed during the experiment. The reason for using that particular serial mouse was 


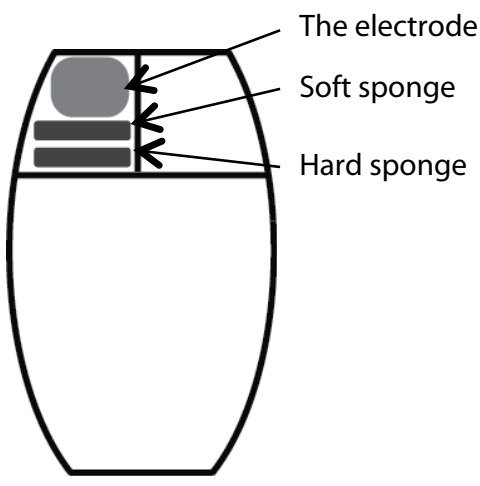

Figure 3. A configuration of the mouse. The electrode and two small pads made with soft sponge and hard sponge were put on the left mouse button.

that it had been studied before and its characteristics were well clarified (Forster \& Forster, 2003; Plant et al., 2003). As is illustrated in Figure 3, the electrode and two small pads were put on the surface of the left mouse button. Both of the pads were made with sponge but were different in hardness. The soft sponge was shrunk by pushing it, whereas the hard one was not. This configuration of the mouse enables both clicking of the button and touching of the electrode simultaneously by the action of clicking the button alone. Another PC was used to record signals from the SMART with a data acquisition (DAQ) unit (Model PCD-320A from Kyowa).

Stimulus. We employed a simple RT paradigm for this experiment. The target stimulus we used was a white circle of 1-cm diameter. It was presented in the center of the display, whose background was set to black (gray value 0 ) and a resolution of $800 \times 600$ pixels. Along with the target stimulus, a white $1 \times 1 \mathrm{~cm}$ square was positioned beneath the $\mathrm{S} 7565$ photodiode to detect the target onset.

Software to conduct this experiment was developed using MS Visual Basic 2005 (VB2005) and DirectX 9.0. The time intervals were controlled and measured by a high-resolution timer of the computer and were programmed with Win32 API of QueryPerformanceFrequency and QueryPerformanceCounter (Xie, Yang, Yang, \& He, 2005).

Procedure. Before the experiment, the brightness and the contrast of the displays were roughly adjusted so that gray values from 0 (black) to 64 were judged black and those from 96 to 255 (white) were judged nonblack at the position beneath the $\mathrm{S} 7576$ photodiode on the screen by the SMART. A trial began by presenting a plain black screen for $600 \mathrm{msec}$, followed by the target stimulus. After recognizing the white circle, the first author pressed the left mouse button. This trial was repeated 20 times for the experiment. The same experiment was done 4 times under different combinatorial conditions of OS (Windows XP or Windows Vista) and displays (CRT or LCD). During the experiment, signals from the photodiode and the electrode, the status signal from the Counter16, and timing signals of detecting stimulus onset and touch by the ADC10 and the CSD, respectively, were recorded by using the DAQ. For this purpose, we used four channels of the DAQ. The sampling rate was set to $5 \mathrm{KHz}$, the maximum rate of the DAQ, for all channels, and the resolution was 12 bits. The signal from the Counter 16 and the timing signals were observed at pins of the PSoCs by adding program codes in the firmware. We also measured two RTs, referred to as RT1 and RT2, and Ta for each trial (see Figure 2). RT1 was the elapsed time between the beginning of presentation of the target stimulus on the display after the latest flip instruction of DirectDraw surface and the completion of registration of the mouse click in the computer measured by the QueryPerformanceCounter function. RT2 was the elapsed time between starting and stopping the Counter16 with a $10-\mathrm{KHz}$ clock from the SMART. Ta was the elapsed time between the arrival of the request from the computer to the dongle and the start of the Counter16.

\section{Results}

Figure 4 shows the changes in signals from stimulus onset to stopping the Counter 16 observed on one of the trials under the condition of Windows XP and LCD. The signal from the photodiode was amplified 20 times. After the actual stimulus onset, the signal from the photodiode gradually increased. Then the onset was detected by using the ADC10 on the expansion card, but it was delayed from the actual onset. After that, the Counter16 on the dongle and the CSD on the expansion card were started. Their signals then went up to around 1.0 and $3.0 \mathrm{~V}$, respectively. When the CSD detected touch to the electrode, the CSD was stopped, and its signal immediately went down to $0 \mathrm{~V}$. The Counter 16 was stopped after receiving notification of touch from the expansion card. Figure 5 shows the changes in signals from the onset of target stimulus to the end of the trial, observed on one of the trials under the condition of Windows XP and LCD (Figure 5A) and on another trial under the condition of Windows XP and CRT (Figure 5B). The signal from the photodiode increased along with repeated local ups and downs in the signal after the stimulus onset. The local ups and downs were slight with the LCD display but large with the CRT display. The signal then continuously decreased. Similar changes in signals, as shown in Figures 4 and 5, were observed on all the trials under all four conditions.

We analyzed the data from the signals recorded in the DAQ and measured (1) time Tb between the beginning of increase in signal from the photodiode after the stimulus onset and starting the Counter16, (2) time Tc between the detection of touch and stopping the Counter16, (3) time Td between the detection of touch and the beginning of continuous decrease in the signal (not part of the ups and downs) from the photodiode, and (4) time Te between the completion of mouse click registration in a trial and the beginning of presenting a plain black screen on the next trial. Under the condition of using the CRT display, the beginning of continuous decrease in the signal from the photodiode, not part of the ups and downs, could not be identified clearly, but it could be in between points $\mathrm{P}$ and $\mathrm{Q}$, as is shown in Figure 5B. Point $\mathrm{Q}$ was determined in such a way that its signal level was the same as at point $R$, the point at which the previous local minimum in signal level was observed. We considered that the average of Td for the CRT display was estimated at the midpoint of $\mathrm{P}$ and $\mathrm{Q}$. Table 1 shows the means and the standard deviations of measured times of $\mathrm{Ta}, \mathrm{Tb}, \mathrm{Tc}$, $\mathrm{Td}$, and $\mathrm{Te}$, calculated times of $\mathrm{Ta}-\mathrm{Tb}, \mathrm{Tb}-\mathrm{Tc}$, and Td - Te, two times of RT1 - RT2, according to RT2 measured by the SMART or the DAQ, and their difference. The estimated timing error in measuring RT by the SMART and the computer are indicated in Table 1. A Kruskal-Wallis test for the measured times and the calculated times was performed across the four conditions, and a Scheffé post hoc test was then used to compare pairs of the four conditions to clarify the influence of the conditions of OS and display. We used R Version 2.9.1, an open-source statistical language and data analysis tool, for the analyses (R Development Core Team, 2009). The results are also shown in Table 1. 


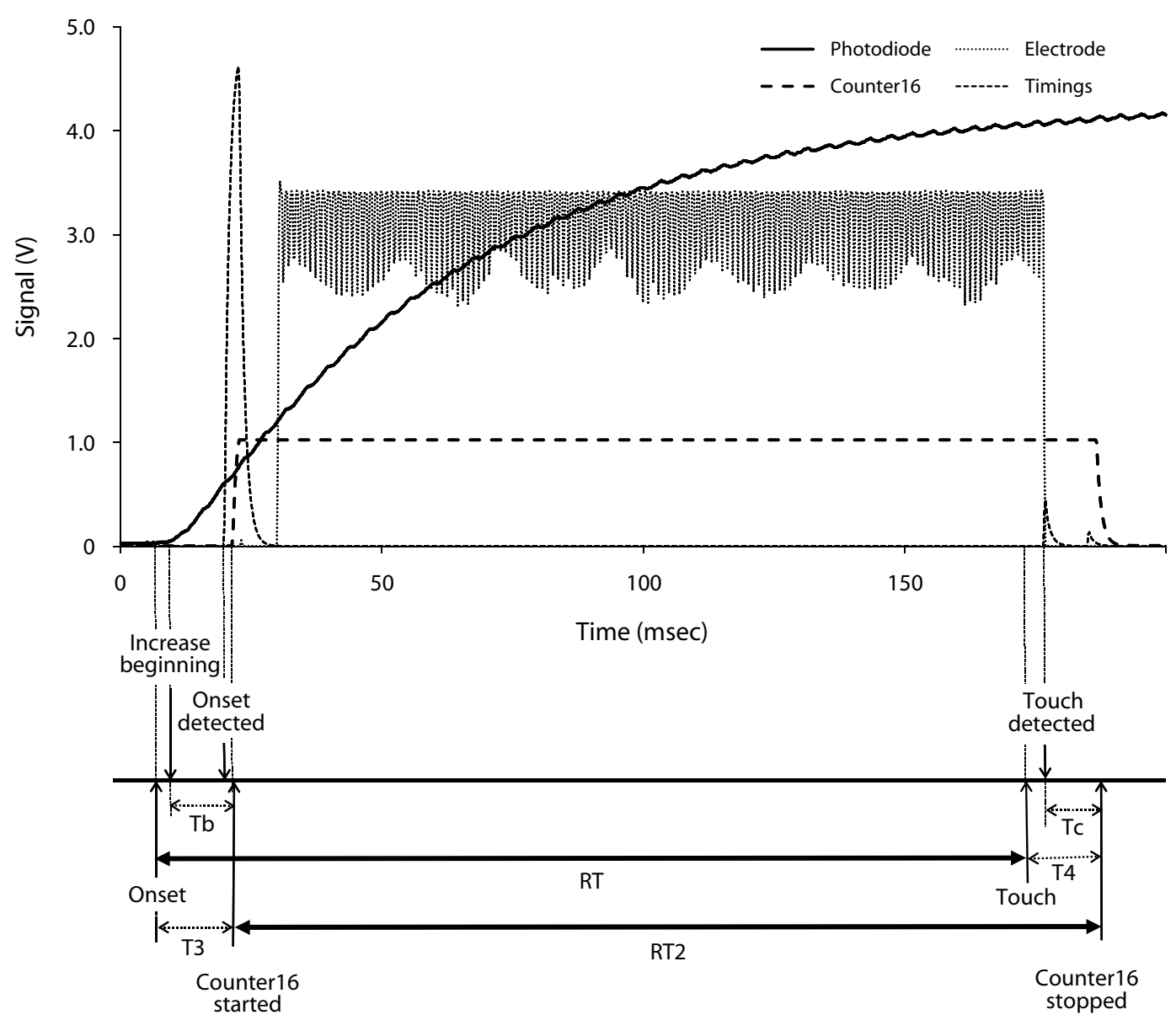

Figure 4. Changes in signals from stimulus onset to stopping Counter16, observed on one of the trials under the condition of Windows XP and LCD display.

\section{Discussion}

Timing accuracy of the SMART. As is shown in Figure 4, the time difference between $\mathrm{T} 3$ and $\mathrm{Tb}$ is the difference between the actual stimulus onset and the beginning of the photodiode's signal increase. It is obvious that the stimulus onset was the direct cause of the continuous increase of the signal level. Due to the characteristics of the photodiode, the signal level goes up immediately after detecting light. This implies that the time difference $\mathrm{T} 3-\mathrm{Tb}$ is very short, and then $\mathrm{Tb}$ could approximate T3. The CSD determines touch only if it stays touch for a predefined number of successive scan cycles. The number was set to 3 , the default value, and the scan cycle was $1.01 \mathrm{msec}$ for the current setting of the CSD. So, the time for determining touch was the sum of the waiting time for the next scan and the time for the three successive scans. The average waiting time was estimated as half of the scan cycle. Therefore, the timing error in RT2 measurement by the SMART, T3 - T4, could be $\mathrm{Tb}-(\mathrm{Tc}+3.5)$, on average. The means and the standard deviations of timing error measured by the SMART under the four conditions are shown in Table 1. The timing error ranged from -0.9 to $0.9 \mathrm{msec}$. As is shown in Table 1, there was a time difference in RT1 - RT2 measured by the SMART and by the DAQ for each condition. This difference was due to the difference in RT2 measured by the two devices. The average of the difference was the same, $1 \mathrm{msec}$, over all the conditions. The accuracy of the clock used in the SMART might cause this difference. By adding this difference to the timing error $\mathrm{T} 3-\mathrm{T} 4$, the overall timing error of the SMART ranged from 0.1 to $1.9 \mathrm{msec}$ (see Table 1).

We examined the influence of jitter and other noise on the SMART to assess its stability. For this purpose, we tested the SMART without the condenser. We found that there were malfunctions in detecting the stimulus onset in that case. However, such malfunctions in detecting stimulus onset were never observed with the condenser in place during Experiment 1. We concluded that the condenser worked as part of the low-pass filter and made the SMART stable in detecting the stimulus onset. Regarding the CSD user module, it was designed to have a high immunity to jitter, chattering, and other noise, with the algorithm for detecting touch as explained above.

From the results and the observations, we concluded that the current configuration of SMART was stable in measuring RT2 and that its timing error was less than $2 \mathrm{msec}$, on average. 

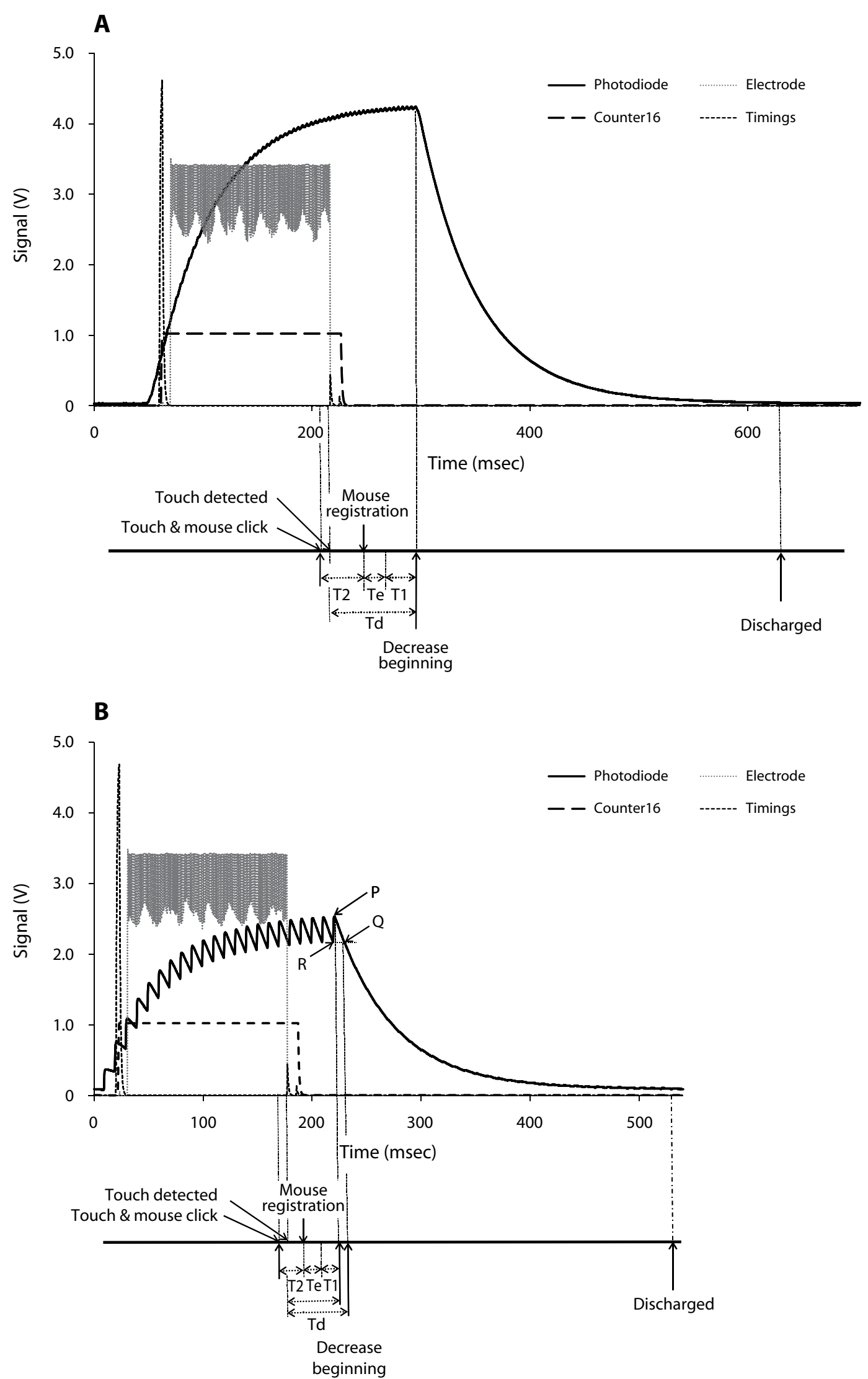

Figure 5. Changes in signals from onset of the target stimulus to the end of the trial, observed on one of the trials under the condition of Windows XP and LCD display (A) and Windows XP and CRT display (B). 
Table 1

\begin{tabular}{|c|c|c|c|c|c|c|c|c|c|c|}
\hline \multirow[b]{3}{*}{ Times } & \multicolumn{4}{|c|}{ Windows Vista } & \multicolumn{4}{|c|}{ Windows XP } & \multirow{2}{*}{\multicolumn{2}{|c|}{ Test $^{\dagger}$}} \\
\hline & \multicolumn{2}{|c|}{ CRT } & \multicolumn{2}{|c|}{ LCD } & \multicolumn{2}{|c|}{ CRT } & \multicolumn{2}{|c|}{ LCD } & & \\
\hline & $M$ & $S D$ & $M$ & $S D$ & $M$ & $S D$ & $M$ & $S D$ & $\chi^{2}$ & $p$ \\
\hline \multicolumn{11}{|l|}{ Measured } \\
\hline $\mathrm{Ta}$ & $12.7^{\mathrm{a}}$ & 0.5 & $21.5^{\mathrm{b}}$ & 0.7 & $12.6^{\mathrm{a}}$ & 0.5 & $21.2^{\mathrm{b}}$ & 0.5 & 61.6 & $<.01$ \\
\hline $\mathrm{Tb}$ & $12.5^{\mathrm{a}}$ & 0.3 & $14.3^{\mathrm{b}}$ & 0.5 & $12.4^{\mathrm{a}}$ & 0.1 & $14.0^{\mathrm{b}}$ & 0.5 & 60.6 & $<.01$ \\
\hline $\mathrm{Tc}$ & 9.8 & 0.5 & 9.8 & 0.3 & 9.7 & 0.5 & 9.9 & 0.4 & 1.89 & .596 \\
\hline $\mathrm{Td}$ & $59.5^{\mathrm{a}}$ & 4.7 & $73.4^{\mathrm{b}}$ & 4.8 & $54.8^{\mathrm{a}}$ & 7.2 & $72.3^{\mathrm{b}}$ & 6.8 & 53.0 & $<.01$ \\
\hline $\mathrm{Te}$ & 11.5 & 3.4 & 11.1 & 5.2 & 12.3 & 6.0 & 13.1 & 5.7 & 1.28 & .732 \\
\hline \multicolumn{11}{|l|}{ Calculated } \\
\hline $\mathrm{Ta}-\mathrm{Tb}$ & $0.2^{\mathrm{a}}$ & 0.3 & $7.3^{\mathrm{b}}$ & 0.4 & $0.2^{\mathrm{a}}$ & 0.5 & $7.2^{\mathrm{b}}$ & 0.4 & 59.6 & $<.01$ \\
\hline $\mathrm{Tb}-\mathrm{Tc}$ & $2.6^{\mathrm{a}}$ & 0.6 & $4.4^{\mathrm{b}}$ & 0.6 & $2.7^{\mathrm{a}}$ & 0.5 & $4.2^{\mathrm{b}}$ & 0.7 & 52.5 & $<.01$ \\
\hline $\mathrm{Td}-\mathrm{Te}$ & $48.0^{\mathrm{a}}$ & 4.6 & $62.3^{\mathrm{b}}$ & 4.0 & $42.5^{\mathrm{a}}$ & 4.3 & $59.1^{\mathrm{b}}$ & 4.1 & 62.5 & $<.01$ \\
\hline \multicolumn{11}{|l|}{ RT1 - RT2 } \\
\hline SMART & 60.3 & 4.1 & 68.9 & 2.1 & 54.7 & 3.5 & 66.2 & 3.7 & & \\
\hline DAQ & 59.3 & 4.1 & 67.9 & 2.1 & 53.7 & 3.5 & 65.3 & 3.7 & & \\
\hline Difference & 1.0 & 0.1 & 1.0 & 0.1 & 1.0 & 0.1 & 1.0 & 0.1 & & \\
\hline \multicolumn{11}{|l|}{ Timing Error } \\
\hline SMART & 0.1 & 0.6 & 1.9 & 0.6 & 0.2 & 0.5 & 1.7 & 0.7 & & \\
\hline Computer & 51.5 & 4.6 & 65.8 & 4.0 & 46.0 & 4.3 & 62.6 & 4.1 & & \\
\hline
\end{tabular}

Note-Means within each row followed by the same letter are not significantly different at the .05 level by Scheffé's pairwise multiple comparison method. †Kruskal-Wallis tests for measured times and calculated times, $n=20$

Timing error in measuring RT1. In Figure 5, the signal from the photodiode continuously decreased (not part of the ups and downs in the signal) after the occurrence of touch and mouse click. In our software, a plain black screen appears after the mouse is clicked. It is reasonable to assume that the onset of a plain black screen in the display was the direct cause of the continuous decrease in the signal. Regarding appearance of the black screen, time $\mathrm{T} 1$ is necessary before the actual onset of the black screen after the completion of the instruction in the computer (see Figure 5). From this and the previous consideration of the time difference between the actual touch and its detection by the CSD, the amount of timing error in measuring RT1 by the computer, T1 + T2, was calculated with $(\mathrm{Td}+3.5)-\mathrm{Te}$. The means and the standard deviations of the timing error for each condition are shown in Table 1. They ranged from 46.0 to $65.8 \mathrm{msec}$.

As is shown in Figure 1 and was noted in the previous discussion on $\mathrm{T} 3, \mathrm{~T} 1$ is estimated as time $\mathrm{Ta}-\mathrm{Tb}$ plus an elapsed time between starting the counter in the computer and the arrival of the request from the computer to the dongle. The elapsed time includes processing time of the USB data transmission in the computer and time to receive data through the USB connection in the dongle. The former depends on other processes running on the computer, but the latter was programmed at 1 -msec interruptions in the firmware of the PSoC. T1 can be considered to be approximately $\mathrm{Ta}-\mathrm{Tb}+1 \mathrm{msec}$ in most of the trials because the instructions for USB transmission in the computer were completed within a short time period. We can suggest that T1 for the CRT and the LCD displays was about 1.2 and $8.2 \mathrm{msec}$, respectively. Now T2 is calculated with $\{(\mathrm{Td}+3.5)-\mathrm{Te}\}-\mathrm{T} 1=(\mathrm{Td}-\mathrm{Te})-(\mathrm{Ta}-\mathrm{Tb})+2.5$. It ranged from 44.8 to $54.4 \mathrm{msec}$. Plant et al. (2003) and
Forster and Forster (2003) investigated the timing error of the MS serial mouse. Their results showed that the error ranged from 40 to $50 \mathrm{msec}$. We concluded that the T2 observed in Experiment 1 concurred well with the previous results obtained by Plant et al. (2003) and Forster and Forster.

Influence of OS and display. From the results of the Kruskal-Wallis test, there were significant differences in $\mathrm{Ta}(p<.01), \mathrm{Tb}(p<.01), \mathrm{Td}(p<.01), \mathrm{Ta}-\mathrm{Tb}(p<$ $.01), \mathrm{Tb}-\mathrm{Tc}(p<.01)$, and $\mathrm{Td}-\mathrm{Te}(p<.01)$, but not in Tc $(p=.596)$ and Te $(p=.732)$. It was then revealed from the result of the Scheffé post hoc analyses that the differences in displays had a greater influence on the times than did the differences in OS. As was discussed above, the timing errors in measuring RT1 and RT2 are calculated with $\mathrm{Td}-\mathrm{Te}+3.5$ and $\mathrm{Tb}-\mathrm{Tc}-3.5$, respectively. The time difference $\mathrm{Td}-\mathrm{Te}$ is very large, and it differs more among the conditions than does $\mathrm{Tb}-\mathrm{Tc}$. From this observation, we could surmise that RT1 measured by the computer would be different among the four conditions, but not for RT2 measured by the SMART.

Perspectives. From this experiment, we conclude that the SMART is stable, reliable, and accurate for measuring RT under different conditions of OS and display. However, we implemented another mode of operating the SMART in order to test it with currently available, well-established systems, such as E-Prime and SuperLab. Flowcharts of the firmware for the PSoCs are shown in Appendix B. A PC and the SMART are used just for measuring RTs. The SMART begins measuring RTs after getting a request from the PC. It then waits for notification of the stimulus onset. After getting the notification, it waits for notification of touch. The Counter16 is stopped, and the RT is returned to the $\mathrm{PC}$ after receiving 


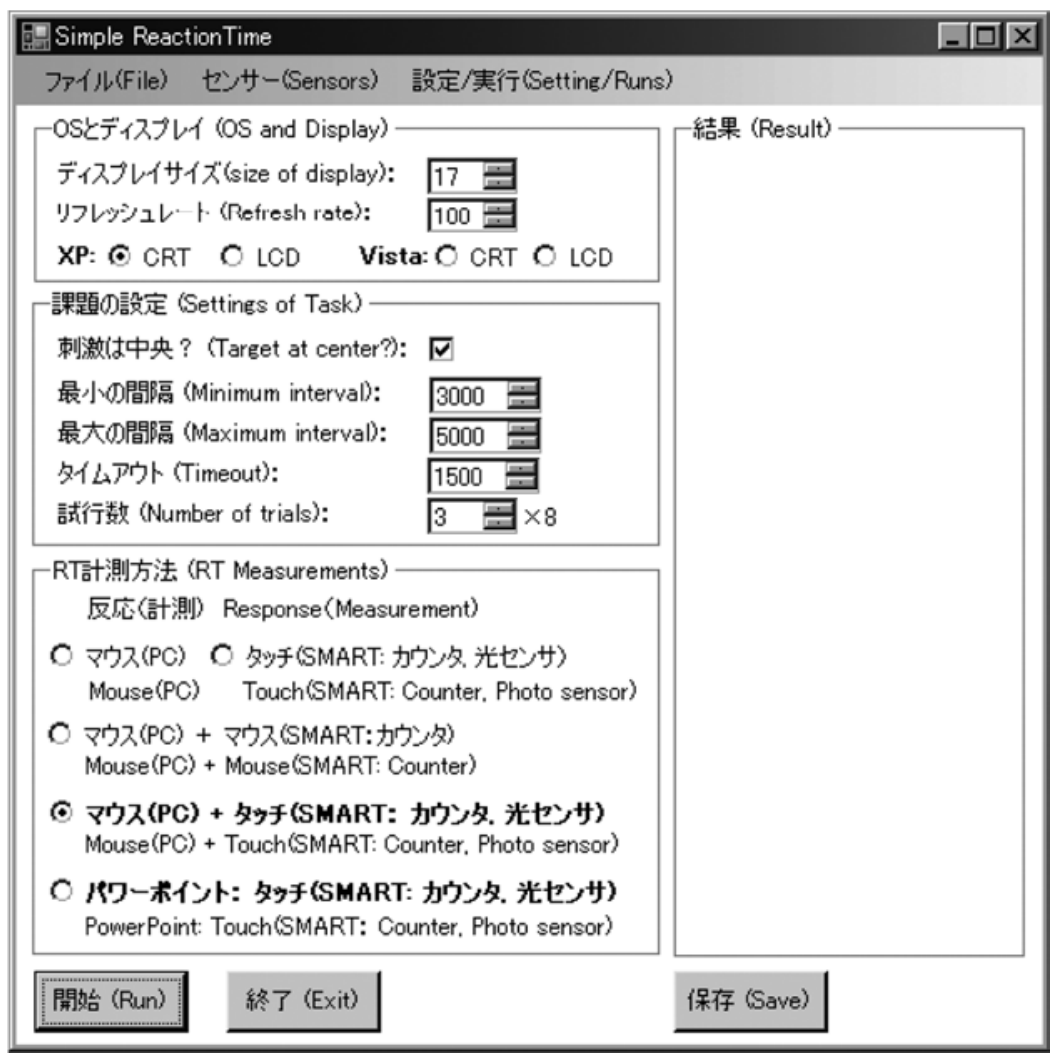

Figure 6. A screenshot of the user interface of software used for the experiments.

the notification of touch; then the SMART repeats the same process until it gets a termination request from the PC. A task for testing the SMART should be prepared by using another system. It is necessary to add a $1 \times 1 \mathrm{~cm}$ white square, at the place underneath the photodiode, to the screen along with the target stimulus and remove it after the onset of the target stimulus. Furthermore, the electrode of the SMART should be put on the response device of the system. By comparing RTs obtained by the SMART and the other system, it is possible to confirm the accuracy of the SMART.

\section{EXPERIMENT 2}

In this experiment, we tested the SMART with a simple RT paradigm under four different combinations of OS and display.

\section{Method}

Participants. We recruited 8 healthy right-handed adults ( 5 women, 3 men), ranging in age from 24 to 42 years ( $M=33.4$ years), from the School of Health Sciences, Sapporo Medical University. All the participants had normal or corrected-to-normal vision.

Apparatus. The apparatus was the same as that in Experiment 1, except that the DAQ was not used. The participants were seated approximately $60 \mathrm{~cm}$ from the display with their heads resting on a chinrest.

Stimulus. We employed a simple RT paradigm. The target stimulus was the same as that in Experiment 1. Two types of trial were employed for this experiment. One served for practicing the use of the mouse and recognizing the center of the screen. This trial began with visual fixation on a $1 \times 1 \mathrm{~cm}$ red square that was presented in place of the target stimulus. The fixation was elicited at randomly varied intervals of 3,000-5,000 msec (uniformly distributed), followed by the target stimulus. The other trial did not employ the visual fixation. Instead of the fixation, a plain black background was presented at the same randomly varied intervals.

Software for conducting this experiment was developed using VB2005 and DirectX 9.0. A screenshot of the user interface is shown in Figure 6. The interval was controlled by using the QueryPerformanceCounter function.

Procedure. Before the experiment, the brightness and the contrast of the displays were roughly adjusted in the same way as in Experiment 1 . This experiment consisted of 2 trials with the fixation, followed by 24 trials without it. The participants were required to click the left mouse button immediately after recognizing the target stimulus. For each trial, RT1 and RT2 were measured. The participants were requested to do the same task four times under the four different combinatorial conditions of OS and display. The order of the four combinations of OS and monitor display was counterbalanced across participants.

\section{Results}

For each task, RTs above 100 msec from 24 trials without the fixation were used to compute the means and the standard deviations. RTs exceeding the mean by three or more standard deviations were then excluded from the analysis as outliers. Table 2 shows the means and the standard deviations of RT1 and RT2 obtained for each participant. The overall means and standard deviations in each condition of OS and display were computed using the 
Table 2

Means and Standard Deviations for Reaction Times (in Milliseconds) of RT1 and RT2 for Each Participant Obtained in Experiment 2

\begin{tabular}{|c|c|c|c|c|c|c|c|c|c|c|c|c|}
\hline \multirow[b]{3}{*}{ Participants } & \multicolumn{6}{|c|}{ Windows Vista } & \multicolumn{6}{|c|}{ Windows XP } \\
\hline & \multicolumn{3}{|c|}{ CRT } & \multicolumn{3}{|c|}{ LCD } & \multicolumn{3}{|c|}{ CRT } & \multicolumn{3}{|c|}{ LCD } \\
\hline & $M$ & $S D$ & $n$ & $M$ & $S D$ & $n$ & $M$ & $S D$ & $n$ & $M$ & $S D$ & $n$ \\
\hline \multicolumn{13}{|l|}{ RT1 } \\
\hline 1 & 317.0 & 57.8 & 24 & 314.4 & 71.0 & 24 & 297.5 & 80.5 & 24 & 287.4 & 52.7 & 23 \\
\hline 2 & 316.1 & 56.0 & 24 & 294.1 & 21.9 & 23 & 272.7 & 45.1 & 23 & 346.1 & 55.6 & 24 \\
\hline 3 & 280.4 & 41.8 & 24 & 339.0 & 68.3 & 24 & 267.8 & 30.4 & 24 & 317.1 & 32.2 & 23 \\
\hline 4 & 288.9 & 26.7 & 23 & 304.5 & 36.6 & 24 & 274.3 & 21.3 & 24 & 313.7 & 27.7 & 24 \\
\hline 5 & 279.1 & 17.4 & 23 & 350.1 & 72.5 & 24 & 264.3 & 15.6 & 23 & 314.6 & 33.7 & 23 \\
\hline 6 & 306.9 & 42.6 & 24 & 314.7 & 54.0 & 23 & 288.8 & 38.0 & 23 & 331.3 & 56.3 & 24 \\
\hline 7 & 305.4 & 76.9 & 24 & 333.3 & 41.9 & 24 & 287.5 & 30.3 & 23 & 315.4 & 49.4 & 23 \\
\hline 8 & 236.8 & 24.3 & 24 & 264.6 & 19.9 & 23 & 248.9 & 40.1 & 24 & 246.2 & 21.0 & 23 \\
\hline Overall & 291.0 & 51.7 & 190 & 312.4 & 53.0 & 188 & 272.3 & 35.7 & 185 & 311.7 & 54.7 & 190 \\
\hline \multicolumn{13}{|l|}{ RT2 } \\
\hline 1 & 191.2 & 27.0 & 24 & 209.7 & 28.4 & 23 & 196.0 & 28.2 & 24 & 173.4 & 23.6 & 23 \\
\hline 2 & 217.3 & 22.0 & 24 & 200.8 & 21.6 & 24 & 186.0 & 27.3 & 20 & 260.4 & 24.9 & 23 \\
\hline 3 & 226.2 & 30.7 & 24 & 216.9 & 34.7 & 24 & 213.2 & 33.4 & 24 & 246.2 & 30.1 & 23 \\
\hline 4 & 220.1 & 30.4 & 23 & 205.6 & 29.6 & 24 & 214.7 & 22.0 & 24 & 226.8 & 37.4 & 24 \\
\hline 5 & 231.3 & 17.8 & 23 & 230.8 & 22.2 & 23 & 212.3 & 15.4 & 23 & 237.0 & 23.8 & 23 \\
\hline 6 & 235.3 & 33.8 & 24 & 221.7 & 39.0 & 24 & 214.4 & 33.9 & 24 & 219.8 & 37.5 & 24 \\
\hline 7 & 180.9 & 28.8 & 24 & 219.2 & 26.4 & 24 & 195.9 & 13.5 & 24 & 219.7 & 36.2 & 23 \\
\hline 8 & 171.6 & 21.9 & 24 & 178.4 & 17.0 & 23 & 171.8 & 20.7 & 23 & 167.5 & 18.7 & 24 \\
\hline Overall & 209.1 & 35.5 & 190 & 209.6 & 30.2 & 188 & 200.7 & 28.7 & 186 & 221.2 & 47.2 & 190 \\
\hline
\end{tabular}

same manner of excluding outlier RTs from the analysis (see Table 2).

A Friedman test was performed on the means of RT1 and RT2 to clarify influence of the conditions of OSs and displays. There was a significant difference in RT1 among the conditions $(p<.05)$, but not in RT2 $(p=.256)$. A Scheffé post hoc analysis showed that there was a significant difference between the combinatorial conditions of the Windows Vista and LCD display and the Windows XP and CRT display $(p<.05)$.

The overall means of RT1 and RT2 ranged from 272.3 to $312.4 \mathrm{msec}$ and from 200.7 to $221.2 \mathrm{msec}$, respectively. The differences in the overall means between RT1 and RT2 for all conditions ranged from 71.6 to $102.8 \mathrm{msec}$.

\section{Discussion}

The results of a Friedman test on the means of RT1 and RT2 were what we anticipated in Experiment 1. The results of Scheffe's test were consistent with the fact that the difference in RT1 - RT2 in Experiment 1 was largest between the conditions of Windows Vista and LCD and Windows XP and CRT.

The overall means of RT2 in each condition seem well suited for the participants in terms of age and gender, because it is known that RTs for young people range from 180 to $200 \mathrm{msec}$ but are affected by age and gender (Kosinski \& Cummings, 1999). The differences in the overall means between RT1 and RT2 were larger than those obtained in Experiment 1, and this might be due to timing differences in touch and mouse click. Although the mouse and the electrode were configured to get simultaneous click and touch by one action of left button click, the touch might have occurred prior to the click, because the participants were not well trained with the configuration.

\section{EXPERIMENT 3}

In this experiment, we used MS PowerPoint with the SMART to present visual stimuli and clarified its timing accuracy with the same simple RT paradigm as that employed in Experiment 2.

\section{Method}

Participants. The participants were the same as those in Experiment 2.

Apparatus. The apparatus was the same as that in Experiment 2, except that the MS serial mouse was not connected to the computer. Only the electrode and two pads placed on the left mouse button were used. MS PowerPoint 2007 was used for this experiment.

Stimuli. The same stimuli as those in Experiment 2 were used but were prepared as a file of MS PowerPoint. We made three slides of a black screen, the fixation, and the target stimulus. Software for conducting the experiment was developed using VB2005. The software controlled the sequence and the duration of presentation of the slides of the file.

Procedure. The procedure was the same as that in Experiment 2, except that only RT2 was measured.

\section{Results}

The analysis performed for each task was the same as that in Experiment 2. Table 3 shows the means and the standard deviations of RT2 obtained for each patient and the overall means and standard deviations in each condition of OS and display.

A Friedman test on the mean RTs was performed. As a result, there was no significant difference among the conditions $(p=.369)$. The overall means of $\mathrm{RT} 2$ ranged from 202.0 to $213.2 \mathrm{msec}$.

We performed a paired-samples $t$ test on the means of RT2 in all the conditions obtained from Experiments 2 and 3 together. The result was that no significant differ- 
Table 3

Means and Standard Deviations for Reaction Times (in Milliseconds) of RT2 for Each Participant Obtained in Experiment 3

\begin{tabular}{|c|c|c|c|c|c|c|c|c|c|c|c|c|}
\hline \multirow[b]{3}{*}{ Participants } & \multicolumn{6}{|c|}{ Windows Vista } & \multicolumn{6}{|c|}{ Windows XP } \\
\hline & \multicolumn{3}{|c|}{ CRT } & \multicolumn{3}{|c|}{$\mathrm{LCD}$} & \multicolumn{3}{|c|}{ CRT } & \multicolumn{3}{|c|}{ LCD } \\
\hline & $M$ & $S D$ & $n$ & $M$ & $S D$ & $n$ & $M$ & $S D$ & $n$ & $M$ & $S D$ & $n$ \\
\hline 1 & 181.2 & 25.5 & 23 & 198.9 & 33.3 & 24 & 204.8 & 22.7 & 23 & 196.8 & 28.2 & 23 \\
\hline 2 & 215.4 & 33.5 & 24 & 194.5 & 26.4 & 24 & 205.7 & 22.1 & 23 & 240.6 & 43.8 & 23 \\
\hline 3 & 231.0 & 34.0 & 23 & 230.4 & 31.1 & 24 & 238.6 & 39.4 & 24 & 233.4 & 43.2 & 24 \\
\hline 4 & 210.6 & 24.8 & 24 & 197.1 & 31.5 & 23 & 208.7 & 27.0 & 23 & 215.7 & 32.2 & 24 \\
\hline 5 & 235.0 & 26.4 & 24 & 217.4 & 29.7 & 24 & 218.8 & 28.5 & 24 & 228.4 & 17.7 & 23 \\
\hline 6 & 226.9 & 28.7 & 24 & 201.7 & 20.1 & 24 & 235.4 & 26.4 & 23 & 206.1 & 26.0 & 24 \\
\hline 7 & 195.7 & 17.9 & 24 & 204.7 & 19.8 & 23 & 189.3 & 31.1 & 23 & 183.7 & 20.7 & 24 \\
\hline 8 & 171.2 & 12.5 & 24 & 173.6 & 16.0 & 23 & 188.7 & 21.7 & 24 & 173.2 & 20.9 & 24 \\
\hline Overall & 207.6 & 32.8 & 189 & 202.0 & 29.6 & 189 & 213.2 & 36.1 & 190 & 209.5 & 36.3 & 190 \\
\hline
\end{tabular}

ence existed between the RTs obtained from the two experiments $(p=.474)$.

\section{Discussion}

To our knowledge, this is the first experiment in which MS PowerPoint has been used to measure RTs with millisecond accuracy. Our experiments confirmed that there is no significant difference in RTs obtained by using DirectX technology with the SMART versus using the PowerPoint file with the SMART.

\section{CONCLUSIONS}

In this study, we explained the SMART system and showed its effectiveness for measuring RT to visual stimuli. We tested the SMART on Windows XP and Vista platforms because of their popularity. The SMART, however, can be used on any platforms of modern computers that support devices of USB HID class. By connecting to CapSense of the PSoC, two or more electrodes can be added to the SMART, and materials and shapes of the electrodes can also be changed. This means that the response device can be configured flexibly according to participants' physical capabilities. Our target participants are mainly young children and elderly people, and it is difficult for some or perhaps all of them to properly click the mouse button. It is true that we do not need millisecond accuracy in clinical settings; 10 -msec accuracy is enough for measuring RT. However, a touch device is indispensable for those who cannot click the mouse button. We are confident that in terms of its stability, accuracy, size, cost, and user friendliness, the SMART is a simple and practical solution for measuring RT, especially in clinical applications. Our approach of using standard office software to present visual stimuli on the display may lead to advances in research and practice in this field.

However, use of the SMART also imposes certain restrictions. From Figure 5, it is obvious that the SMART can be used only after the condenser is fully discharged. This means that a sufficient time interval between onsets of successive target stimuli has to be ensured in order to make the SMART work. In addition, the SMART ensures accurate measurement only of the interval between a visual stimulus onset and a response. Other timing issues and supporting auditory stimuli are not taken into account by the current version of SMART. Resolving these issues could make the SMART practical not only for simple RT paradigms, but for other RT paradigms as well. Notwithstanding these restrictions, we are certain that the SMART is able to provide both researchers and health professionals with new ways of using RT paradigms in their work.

\section{AUTHOR NOTE}

We thank two reviewers for very valuable and helpful comments on the first draft of this article. Especially, we thank Hans Strasburger, one of the reviewers, for his kindness in providing us with his language corrections to the first draft. Correspondence concerning this article should be addressed to T. Ohyanagi, Center for Medical Education, Sapporo Medical University, South 1, West 17, Chuo-ku, Sapporo, 060-8556, Japan (e-mail: ohyanagi@sapmed.ac.jp).

\section{REFERENCES}

Cypress Semiconductor Corporation (2007). PSoC FirstTouch Guide. Retrieved July 21, 2009, from www.cypress.com/?docID=9443. De Clercq, A., Crombez, G., Buysse, A., \& Roeyers, H. (2003). A simple and sensitive method to measure timing accuracy. Behavior Research Methods, Instruments, \& Computers, 35, 109-115.

Forster, K. I., \& Forster, J. C. (2003). DMDX: A Windows display program with millisecond accuracy. Behavior Research Methods, Instruments, \& Computers, 35, 116-124.

Hamamatsu Photonics K. K. (2001). Photo IC diode S7565, S7805-10. Retrieved July 21, 2009, from http://sales.hamamatsu.com/assets/pdf/ parts_S/S7565_S7805-10.pdf.

Häusler, J., Sommer, M., \& Chroust, S. (2007). Optimizing technical precision of measurement in computerized psychological assessment on Windows platforms. Psychology Science, 49, 116-131.

Kosinski, B., \& Cummings, J. (1999). The scientific method: An introduction using reaction time. In S. J. Karcher (Ed.), Tested studies for laboratory teaching, 20, 63-84. Retrieved July 21, 2009, from www .ableweb.org/volumes/vol-20/3-kosinski.pdf.

Krantz, J. H. (2000). Tell me, what did you see? The stimulus on computers. Behavior Research Methods, Instruments, \& Computers, 32, 221-229.

McKinney, C. J., MacCormac, E. R., \& Welsh-Bohmer, K. A. (1999). Hardware and software for tachistoscopy: How to make accurate measurements on any PC utilizing the Microsoft Windows operating system. Behavior Research Methods, Instruments, \& Computers, 31, 129-136.

Plant, R. R., Hammond, N., \& Turner, G. (2004). Self-validating presentation and response timing in cognitive paradigms: How and why? Behavior Research Methods, Instruments, \& Computers, 36, 291-303.

Plant, R. R., Hammond, N., \& Whitehouse, T. (2003). How choice of mouse may affect response timing in psychological studies. Behavior Research Methods, Instruments, \& Computers, 35, 276-284. 
R DeVelopment CoRe TeAm (2009). $R$ : A language and environment for statistical computing. Vienna: R Foundation for Statistical Computing.

SHimizU, H. (2002). Measuring keyboard response delays by comparing keyboard and joystick inputs. Behavior Research Methods, Instruments, \& Computers, 34, 250-256.

Wiens, S., Fransson, P., Dietrich, T., Lohmann, P., Ingvar, M., \& Öhman, A. (2004). Keeping it short: A comparison of methods for brief picture presentation. Psychological Science, 15, 282-285.
XIE, S., YANG, Y., YANG, Z., \& HE, J. (2005). Millisecond-accurate synchronization of visual stimulus displays for cognitive research. Behavior Research Methods, 37, 373-378.

\section{NOTE}

1. The software and firmware can be obtained by e-mailing Toshio Ohyanagi at ohyanagi@sapmed.ac.jp.

APPENDIXA

Flowcharts of Firmware Used in the Experiments Running on PSoCs Residing on the Dongle and the Expansion Card

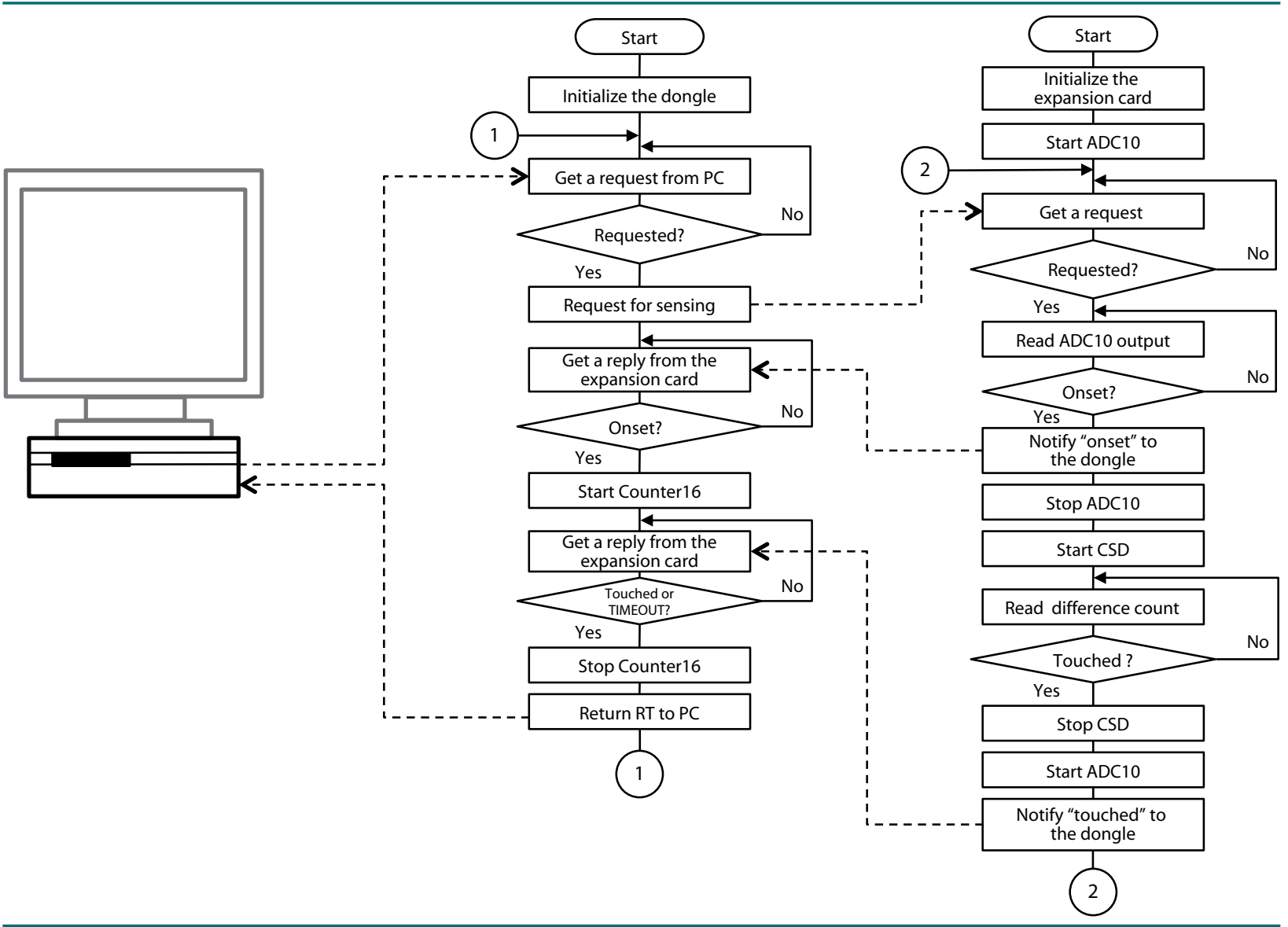




\section{APPENDIX B}

Flowcharts of Firmware on PSoCs Residing on the Dongle and the Expansion Card Running in Another Mode to Measure RTs by the Computer (Stimuli Should Be Presented via Another System)

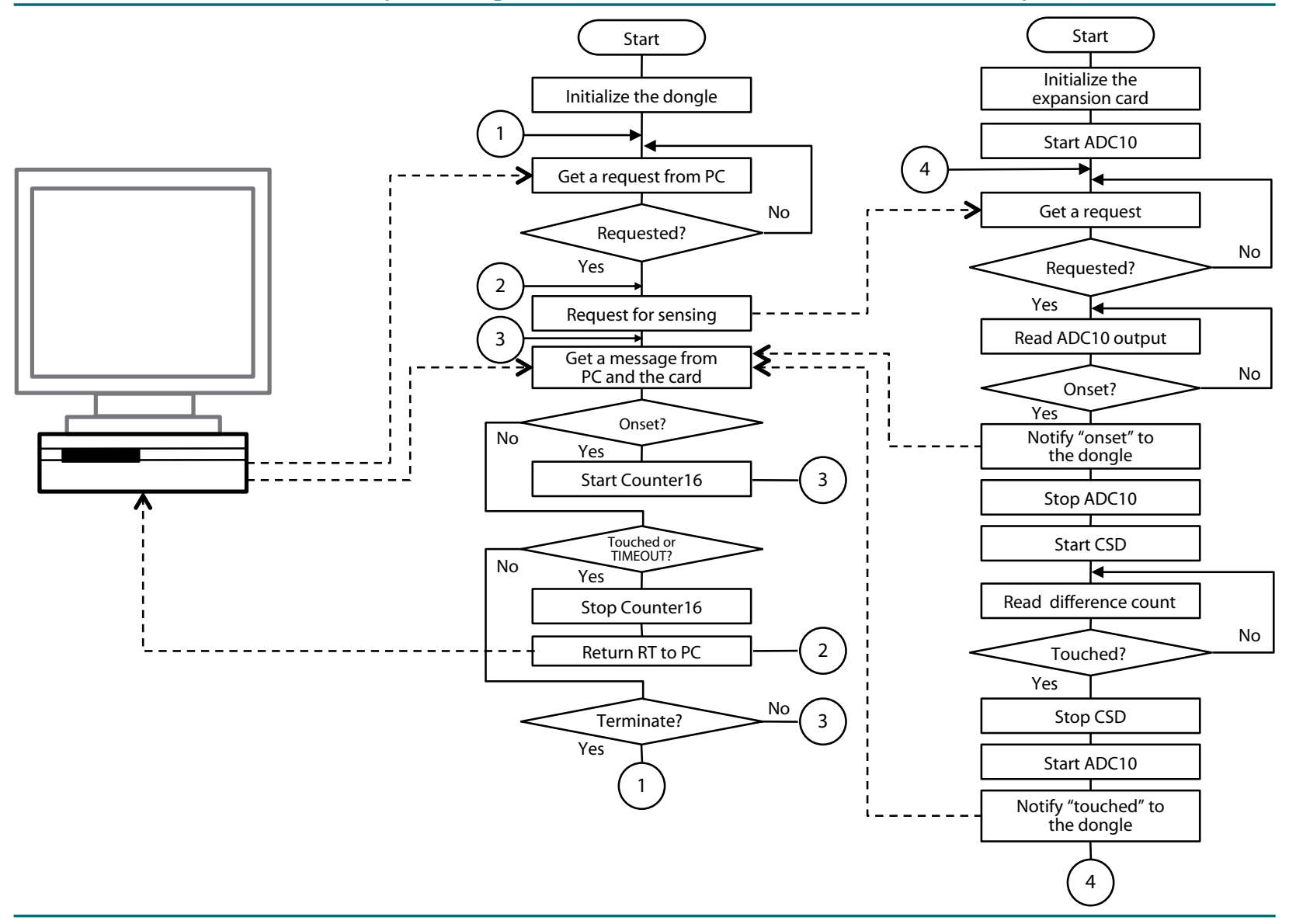

(Manuscript received April 13, 2009;

revision accepted for publication September 30, 2009.) 